\title{
Modeled forest conversion influences humid tropical watershed hydrology more than projected climate change
}

\author{
Taylor Joyal ${ }^{1}$, Alexander Fremier ${ }^{2}$, and Jan Boll ${ }^{2}$ \\ ${ }^{1}$ Northern Arizona University - Flagstaff Mountain Campus \\ ${ }^{2}$ Washington State University
}

December 5, 2021

\begin{abstract}
In the humid tropics, forest conversion and climate change threaten the hydrological function and stationarity of watersheds, particularly in steep terrain. As climate change intensifies, shifting precipitation patterns and expanding agricultural and pastoral land use may effectively reduce the resilience of headwater catchments. Compounding this problem is the limited long-term monitoring in developing countries for planning in an uncertain future. In this paper, we asked which change, climate or land use, more greatly affects stream discharge in humid tropical mountain watersheds? To answer this question, we used the process-based, spatially distributed Soil Moisture Routing model. After first evaluating model performance (Ns $=0.73$ ), we conducted a global sensitivity analysis to identify the model parameters that most strongly influence simulated watershed discharge. In particular, peak flows are most influenced by input model parameters that represent baseflow and shallow subsurface soil pathways while low flows are most sensitive to antecedent moisture, macropore hydraulic conductivity, soil depth and porosity parameters. We then simulated a range of land use and climate scenarios in three mountain watersheds of central Costa Rica. Our results show that deforestation influences streamflow more than altered precipitation and temperature patterns through changes in first-order hydrologic hillslope processes. However, forest conversion coupled with intensifying precipitation events amplifies hydrological extremes, reducing the hydrological resilience to predicted climate shifts in mountain watersheds of the humid tropics. This finding suggests that reforestation can help mitigate the effects of climate change on streamflow dynamics in the tropics including impacts to water availability, flood pulses, channel geomorphology and aquatic habitat associated with altered flow regimes.
\end{abstract}

\section{Hosted file}

Joyal et al_2022_MAIN TEXT.docx available at https://authorea.com/users/449657/articles/ 548145-modeled-forest-conversion-influences-humid-tropical-watershed-hydrology-morethan-projected-climate-change

Table 1. Watershed Characteristics

\begin{tabular}{|l|c|c|c|c|c|}
\hline Watershed & Drainage Area & Elevation Range & Max Slope & Median Slope & Altered Land Cover \\
\hline \hline Gato & $3,340 \mathrm{ha}$ & $755-2,355 \mathrm{~m}$ & $58^{\circ}$ & $31^{\circ}$ & $<0.1 \%$ \\
\hline Atirro & $3,249 \mathrm{ha}$ & $780-1,980 \mathrm{~m}$ & $60^{\circ}$ & $26^{\circ}$ & $2.5 \%$ \\
\hline Platanillo & $2,595 \mathrm{ha}$ & $700-1,940 \mathrm{~m}$ & $56^{\circ}$ & $24^{\circ}$ & $41.0 \%$ \\
\hline
\end{tabular}


Table 2. Input Parameters for Soil Moisture Routing Model

\begin{tabular}{|c|c|c|}
\hline SMR Input Parameter & Source & Value \\
\hline $\begin{array}{l}\text { Precipitation (not in } \\
\text { GSA) }\end{array}$ & $\begin{array}{l}\text { Meteorological station data (ICE), Climate models } \\
\text { (Solomon (2007), Stocker (2014)) }\end{array}$ & See Figure 4 \\
\hline PET (not in GSA) & Hamon PET equation & Calculated ET: $0.16-0.24 \mathrm{~cm} /$ day \\
\hline Crop Coefficient & $\begin{array}{l}\text { General value for humid tropical vegetation } \\
\text { (Allen et al. 1998) }\end{array}$ & 0.8 \\
\hline Soil Depth & $\begin{array}{l}\text { Field sampled and corrected for slope; depth of } \\
\text { hydrologic soil layers A \& B adopted from Hanson } \\
\text { et al. (2004) and Toohey et al. (2018) }\end{array}$ & $\begin{array}{l}\text { Forest: A: } 35 \mathrm{~cm}, \mathrm{~B}: 65 \mathrm{~cm} \\
\text { Coffee: A: } 50 \mathrm{~cm}, \mathrm{~B}: 50 \mathrm{~cm} \\
\text { Sugar Cane: A: } 50 \mathrm{~cm}, \mathrm{~B}: 50 \mathrm{~cm} \\
\text { Pasture: A: } 10 \mathrm{~cm}, \mathrm{~B}: 90 \mathrm{~cm} \\
\text { Roadt: A: } 50 \mathrm{~cm}, \mathrm{~B}: 50 \mathrm{~cm}\end{array}$ \\
\hline Slope (not in GSA) & DEM & Range: $0-62^{\circ}$ \\
\hline$K_{\text {sat }}$ Matrix & $\begin{array}{l}\text { Spaans et al. (1989), Hanson et al. (2004), Toohey } \\
\text { et al. (2018) }\end{array}$ & $\begin{array}{l}\text { Forest: A: } 13.4, B: 8.9 \mathrm{~cm} / \text { day } \\
\text { Coffee: A: } 7.7, \text { B: } 8.9 \mathrm{~cm} / \text { day } \\
\text { Sugar Cane: A: } 3.1, B: 1.7 \mathrm{~cm} / \text { day } \\
\text { Pasture: A: } 2.9, \text { B: } 5.5 \mathrm{~cm} / \text { day } \\
\text { Roadt: A: } 0.05, \text { B: } 0.05 \mathrm{~cm} / \text { day }\end{array}$ \\
\hline$K_{\text {sat }}$ Macropore & $\begin{array}{l}\text { Spaans et al. (1989), Hanson et al. (2004), Toohey } \\
\text { (2012) }\end{array}$ & $\begin{array}{l}\text { Forest: A: } 134.4, \mathrm{~B}: 88.8 \mathrm{~cm} / \text { day } \\
\text { Coffee: A: 76.8, B: } 88.8 \mathrm{~cm} / \text { day } \\
\text { Sugar Cane: A: } 31.2, \mathrm{~B}: 16.8 \\
\mathrm{~cm} / \text { day } \\
\text { Pasture: A: } 28.8, \mathrm{~B}: 55.2 \mathrm{~cm} / \text { day } \\
\text { Roadt: A: } 1.0, \mathrm{~B}: 1.0 \mathrm{~cm} / \text { day }\end{array}$ \\
\hline$\kappa_{\text {sub }}$ & Toohey (2012), Estimated & $\begin{array}{l}\text { Forest: } 84.0 \mathrm{~cm} / \text { day } \\
\text { Coffee: } 84.0 \mathrm{~cm} / \text { day } \\
\text { Sugar Cane: } 1.4 \mathrm{~cm} / \text { day } \\
\text { Pasture: } 4.8 \mathrm{~cm} / \text { day } \\
\text { Roadt: } 1.4 \mathrm{~cm} / \text { day }\end{array}$ \\
\hline $\begin{array}{l}\text { Field Capacity Moisture } \\
\text { Content }\end{array}$ & Toohey (2012) & $\begin{array}{l}\text { Forest: } 28 \% \\
\text { Coffee: } 28 \% \\
\text { Sugar Cane: } 28 \% \\
\text { Pasture: } 18 \% \\
\text { Roadt: } 8 \%\end{array}$ \\
\hline $\begin{array}{l}\text { Porosity (Saturated } \\
\text { Moisture Content) }\end{array}$ & Field sampled; Spaans et al. (1989) & $\begin{array}{l}\text { Forest: A: } 38 \%, \text { B: } 28 \% \\
\text { Coffee: A: } 38 \%, \text { B: } 28 \% \\
\text { Sugar Cane: A: } 38 \%, \text { B: } 28 \% \\
\text { Pasture: A: } 28 \%, \text { B: } 18 \% \\
\text { Roadt: A: } 3.8 \%, \text { B: } 2.8 \%\end{array}$ \\
\hline $\begin{array}{l}\text { Residual Moisture } \\
\text { Content }\end{array}$ & Spaans et al. (1989), Toohey (2012) & All land cover types: $2 \%$ \\
\hline $\begin{array}{l}\text { Wilting Point Moisture } \\
\text { Content }\end{array}$ & Toohey (2012) & $\begin{array}{l}\text { All land cover types: A: } 1.94 \%, B \text { : } \\
1.21 \%\end{array}$ \\
\hline $\begin{array}{l}\text { Max Canopy Storage } \\
\text { Amount } \\
\text { (not in GSA) }\end{array}$ & Welsh et al. (2018); Estimated & $\begin{array}{l}\text { Forest: } 0.2 \mathrm{~cm} \\
\text { Coffee: } 0.1 \mathrm{~cm} \\
\text { Sugar Cane: } 0.05 \mathrm{~cm} \\
\text { Pasture: } 0.05 \mathrm{~cm} \\
\text { Road: } 0.0 \mathrm{~cm}\end{array}$ \\
\hline Rock Content & Field sampled & All land cover types: $10 \%$ \\
\hline Road Area (not in GSA) & Peréz (2009), Aerial imagery & $\begin{array}{l}\text { Atirro: } 7.66 \text { ha; } 0.24 \% \text { cover } \\
\text { Gato: } 0 \text { ha; } 0 \% \text { cover } \\
\text { Platanillo: } 22.98 \text { ha; } 0.89 \% \text { cover }\end{array}$ \\
\hline Recession Constants & Estimated based on gauge data & $a=75 ; b=0.2$ (See equation in text) \\
\hline $\begin{array}{l}\text { Antecedent Moisture } \\
\text { Content }\end{array}$ & Estimated (model equilibrates) & $72 \mathrm{~cm}$ \\
\hline
\end{tabular}

GSA - Global Sensitivity Analysis; tall road parameters estimated

Table 3. Simulation Scenario Matrix

\begin{tabular}{|c|c|c|c|c|}
\hline & Land Use & $\begin{array}{l}\text { Rainfall } \\
\text { Intensity }\end{array}$ & $\begin{array}{l}\text { Temperature (based on } \\
2002 \text { record) }\end{array}$ & $\begin{array}{l}\text { Rainfall Amount (\% of } \\
2002 \text { precipitation) }\end{array}$ \\
\hline Atirro & $\begin{array}{l}\text { - All pasture } \\
\text { - Existing }\end{array}$ & $\begin{array}{l}\cdot \text { High rate } \\
\cdot \text { Existing rate }\end{array}$ & $\begin{array}{l}\cdot \text { Existing Temperature } \\
\cdot+1^{\circ} \mathrm{C} \text { (daily time step only) } \\
\cdot+4{ }^{\circ} \mathrm{C}\end{array}$ & $\begin{array}{l}\cdot 70 \% \\
\cdot 100 \% \\
\cdot 110 \% \\
\end{array}$ \\
\hline Gato & $\begin{array}{l}\text { - All pasture } \\
\text { - Existing }\end{array}$ & $\begin{array}{l}\cdot \text { High rate } \\
\cdot \text { Existing rate }\end{array}$ & & \\
\hline Platanillo & $\begin{array}{l}\cdot \text { All pasture } \\
\cdot \text { Existing } \\
\text { - All forested }\end{array}$ & $\begin{array}{l}\cdot \text { High rate } \\
\cdot \text { Existing rate }\end{array}$ & & \\
\hline
\end{tabular}


Table 4. Gini Importance Values in SMR Sensitivity Analysis

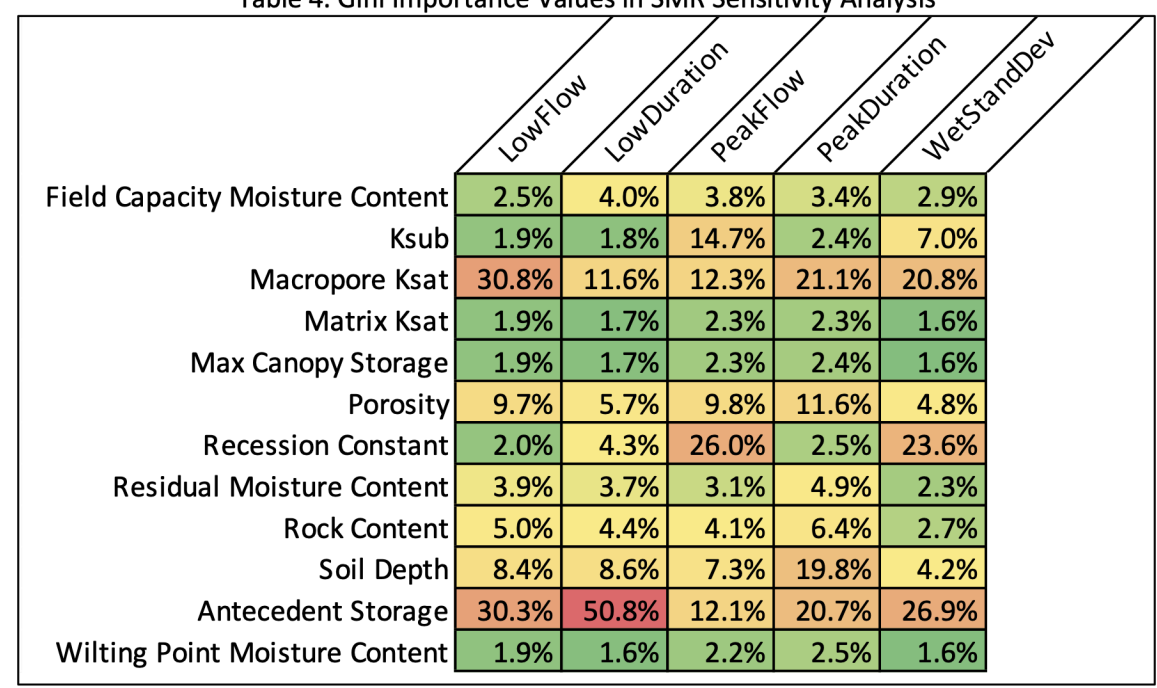

\begin{tabular}{|c|c|c|c|c|c|c|c|c|}
\hline \multicolumn{3}{|c|}{ Atirro Existing } & \multicolumn{3}{|c|}{ Atirro Pasture Conversion } & \multicolumn{3}{|c|}{ Atirro Pasture with Restrictive Layer } \\
\hline \multicolumn{2}{|l|}{ MASS BALANCE } & \multirow{2}{*}{\begin{tabular}{|c|} 
DEFICIT \\
ppt - output
\end{tabular}} & \multicolumn{2}{|c|}{ MASS BALANCE } & \multirow{2}{*}{\begin{tabular}{c|} 
DEFICIT \\
ppt - output
\end{tabular}} & \multicolumn{2}{|l|}{ MASS BALANCE } & \multirow{2}{*}{\begin{tabular}{c|} 
DEFICIT \\
ppt - output
\end{tabular}} \\
\hline ppt input (cm/basin) & $\begin{array}{c}\text { output } \\
\text { (cm/basin) }\end{array}$ & & ppt input (cm/basin) & $\begin{array}{c}\text { output } \\
\text { (cm/basin) }\end{array}$ & & ppt input (cm/basin) & $\begin{array}{c}\text { output } \\
\text { (cm/basin) }\end{array}$ & \\
\hline 503.5 & 480.8 & 22.7 & 503.5 & 496.3 & 7.2 & 503.5 & 496.3 & 7.2 \\
\hline Water Balance Term & $\%$ & $\mathrm{~cm}$ & Water Balance Term & $\%$ & $\mathrm{~cm}$ & Water Balance Term & $\%$ & $\mathrm{~cm}$ \\
\hline Precipitation & $100.0 \%$ & 503.5 & Precipitation & $100.0 \%$ & 503.5 & Precipitation & $100.0 \%$ & 503.5 \\
\hline Evapotranspiration & $11.6 \%$ & 58.4 & Evapotranspiration & $12.3 \%$ & 61.8 & Evapotranspiration & $12.3 \%$ & 61.8 \\
\hline Baseflow & $76.5 \%$ & 385.3 & Baseflow & $74.4 \%$ & 374.8 & Baseflow & $40.8 \%$ & 205.6 \\
\hline Saturation Excess Runoff & $7.4 \%$ & 37.1 & Saturation Excess Runoff & $11.9 \%$ & 59.8 & Saturation Excess Runoff & $45.5 \%$ & 229.0 \\
\hline Storage & $4.5 \%$ & 22.7 & Storage & $1.4 \%$ & 7.2 & Storage & $1.4 \%$ & 7.2 \\
\hline
\end{tabular}
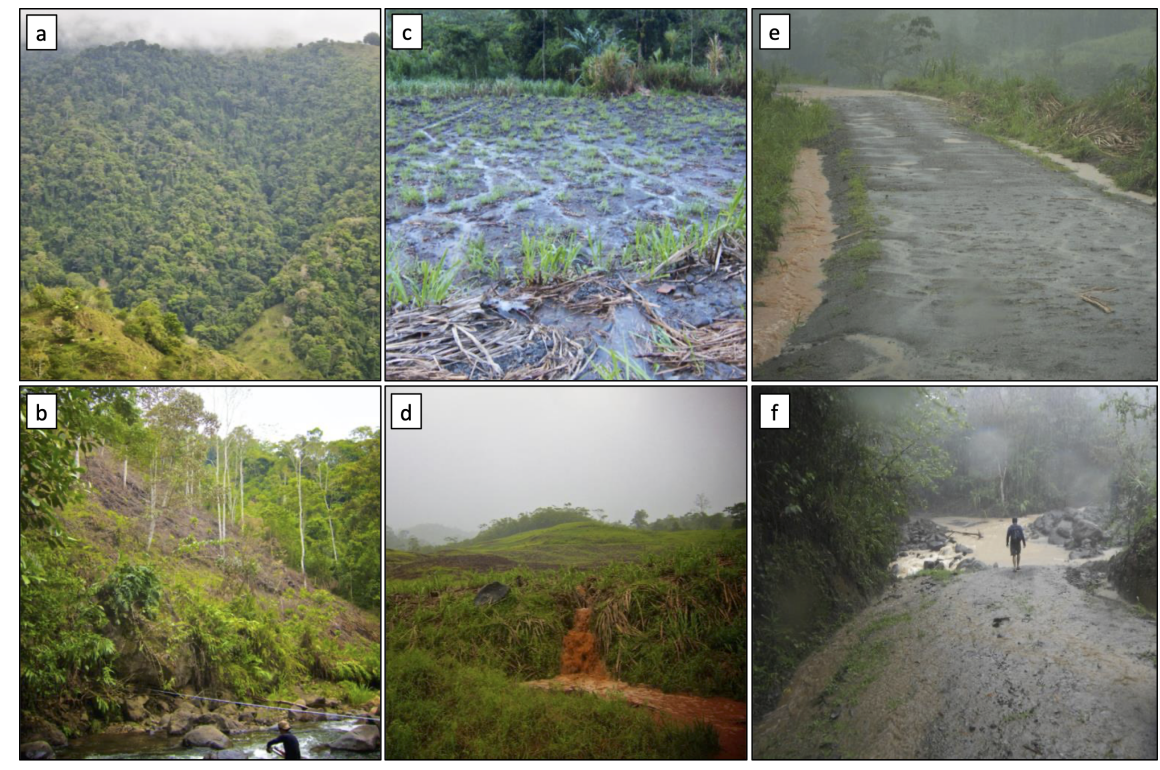

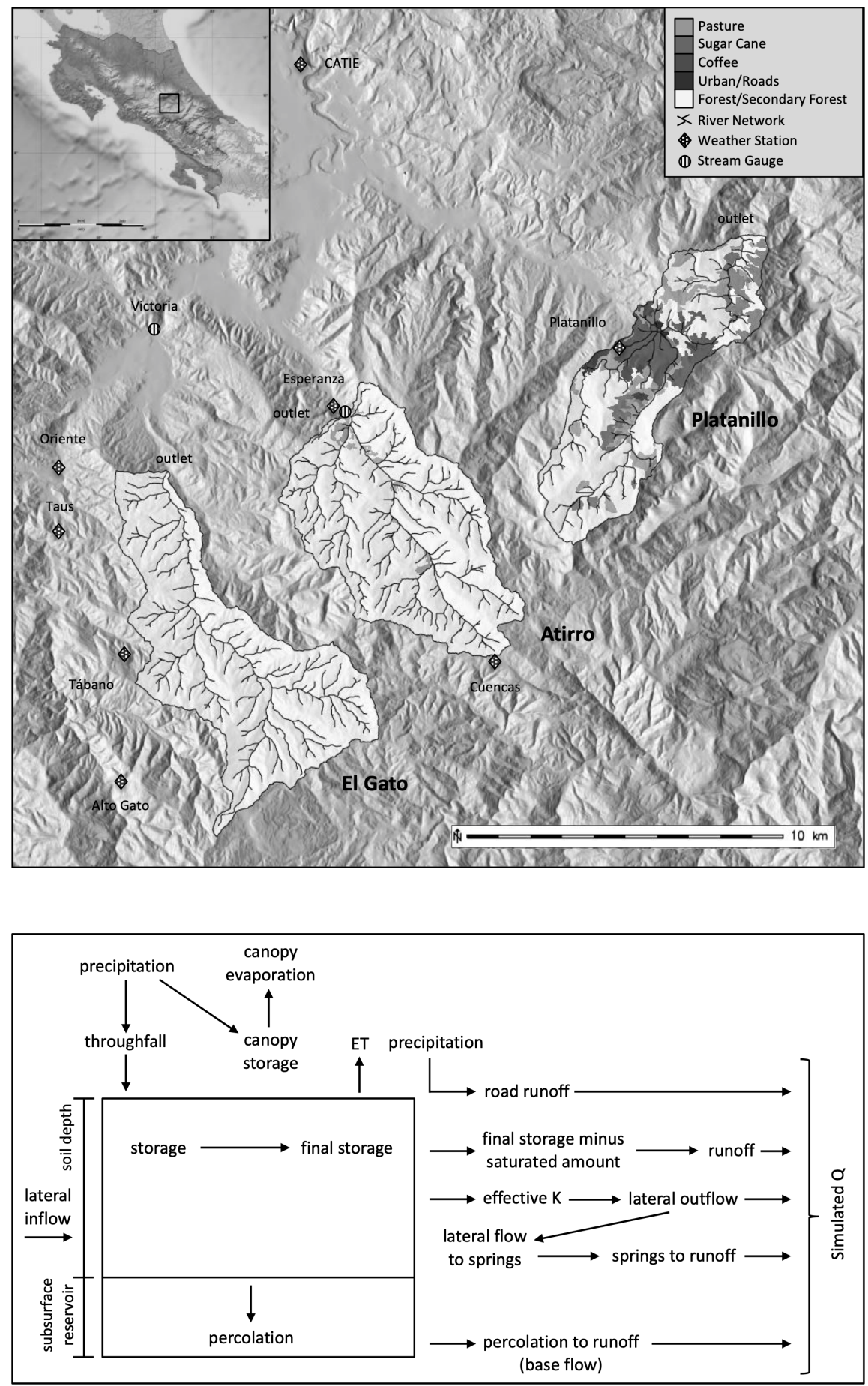

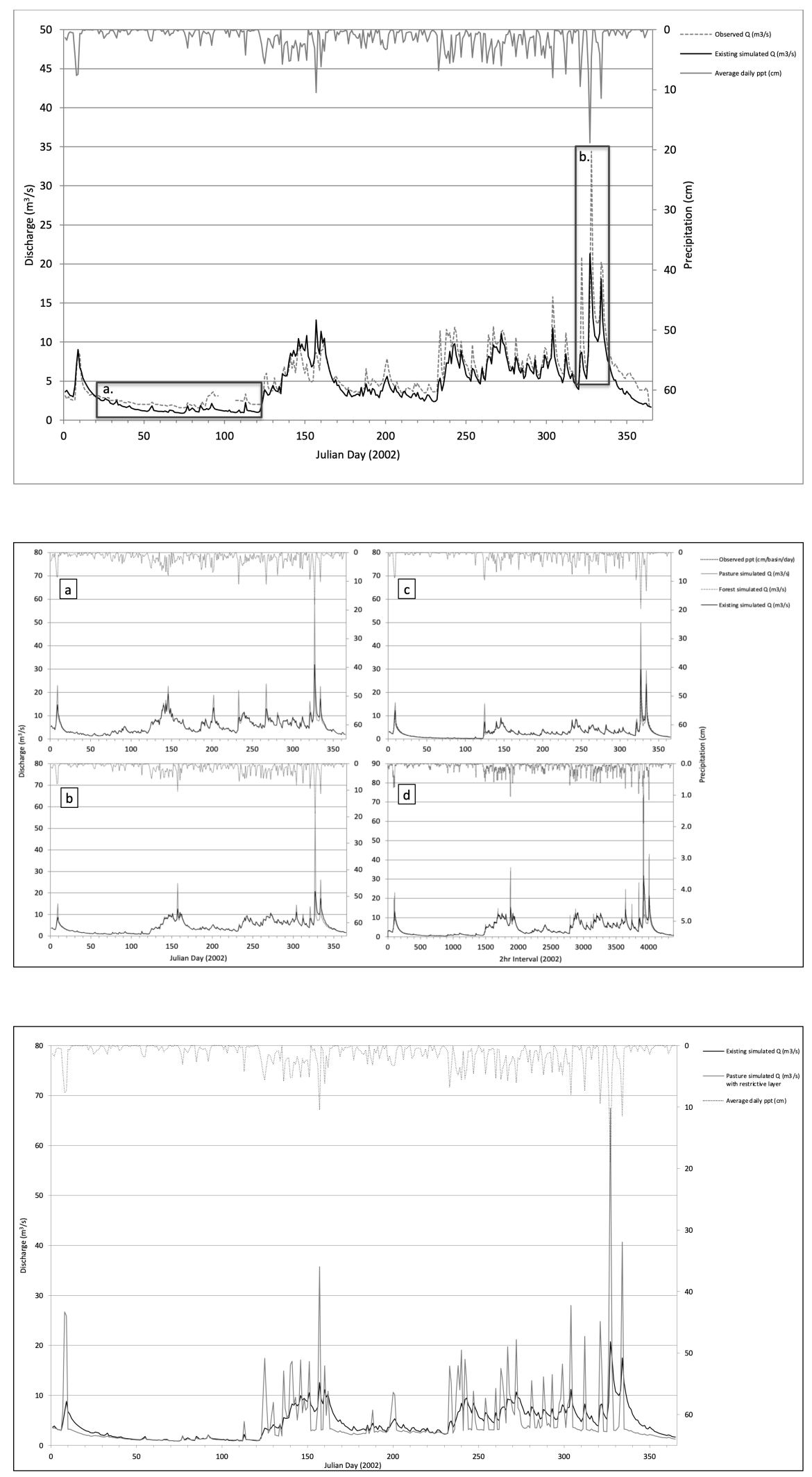

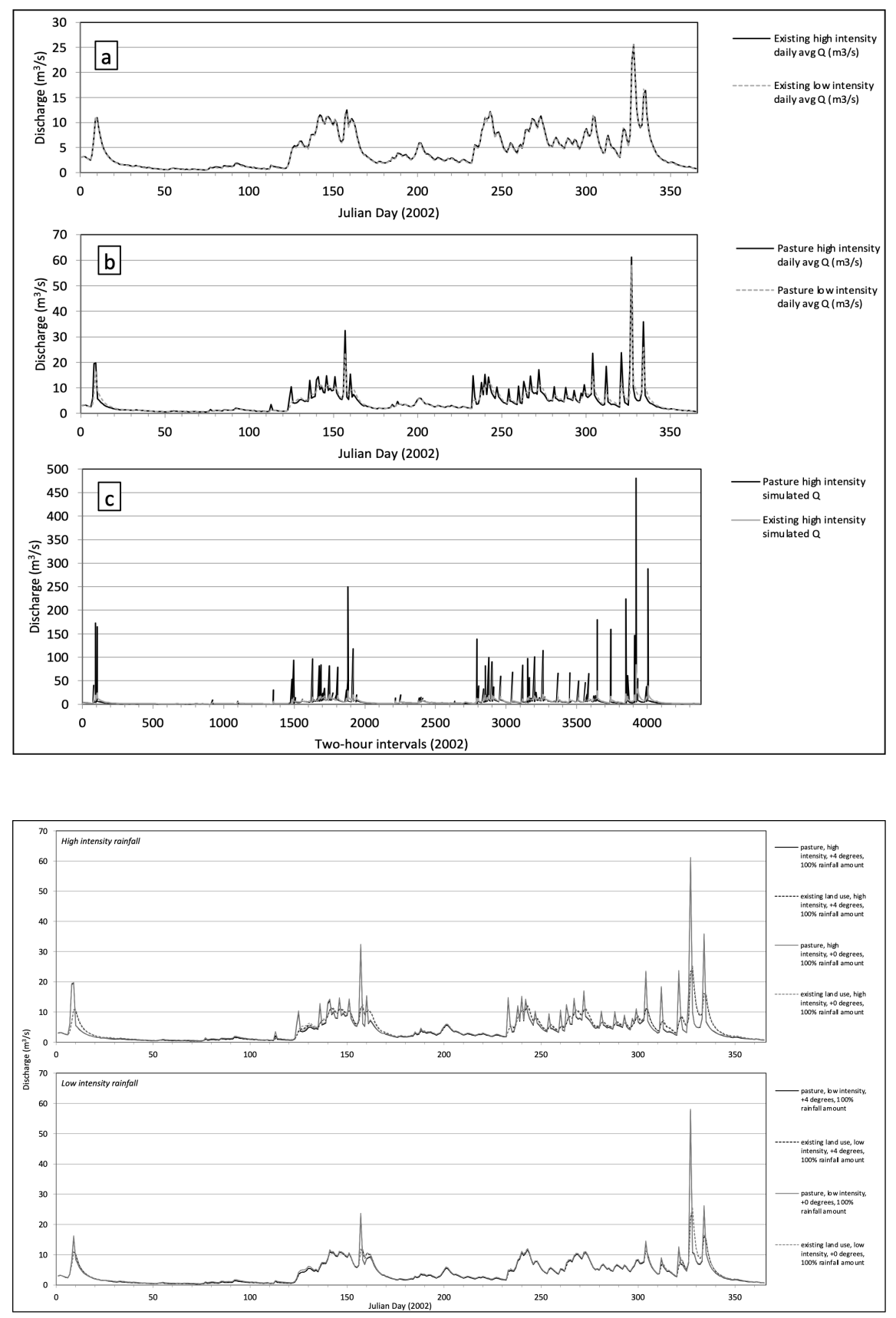


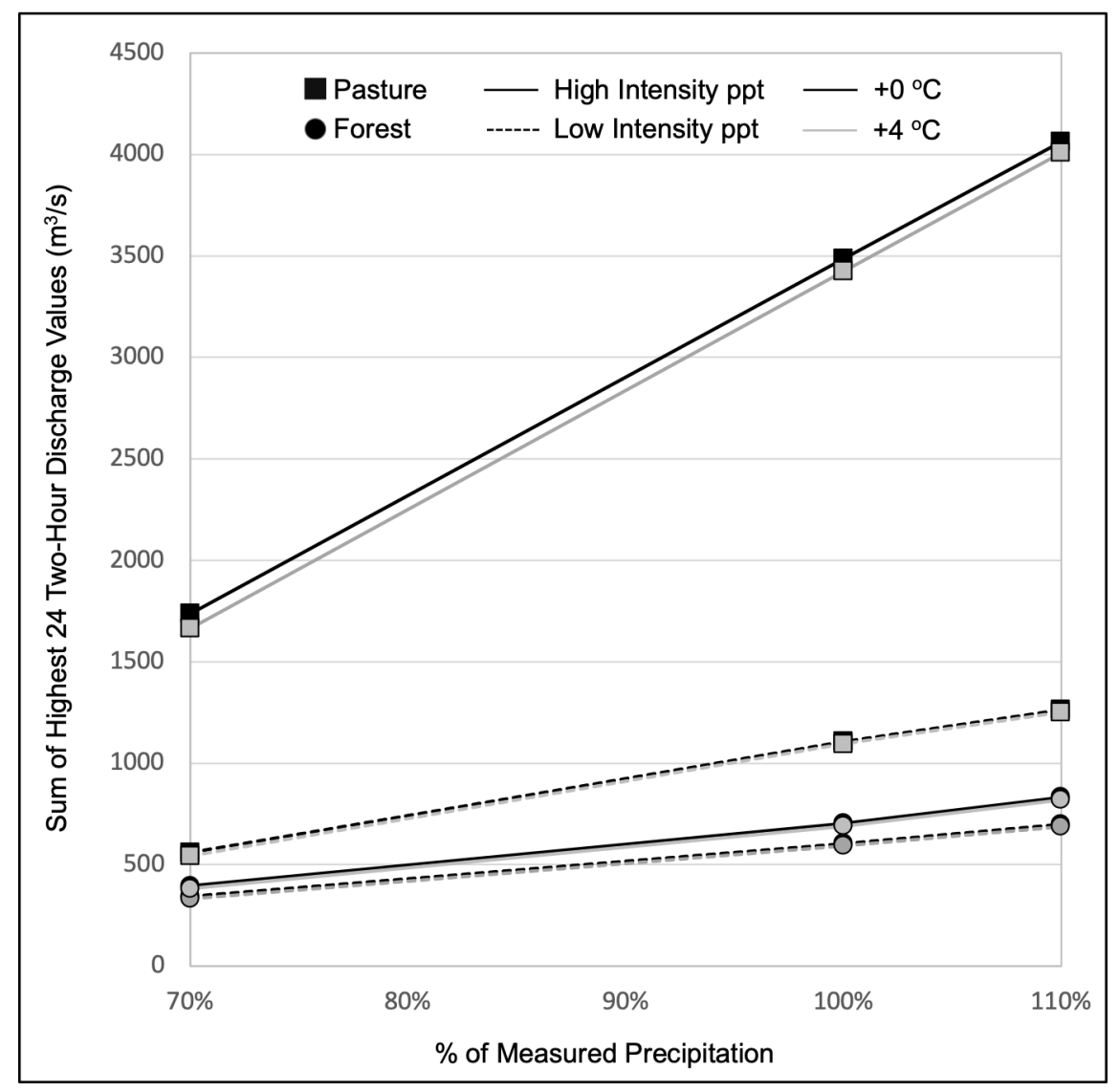

